\title{
Biomechanical Comparison between Stand-alone Interbody Cages and their Benefits over Posterior Screw Fixation.
}

\author{
Andrea Calvo-Echenique, José Cegoñino, Amaya Pérez-del Palomar \\ Group of Biomaterials (GBM) \\ Instituto de Investigación en Ingeniería de Aragón (I3A) \\ Universidad de Zaragoza, Mariano Esquillor s/n, 50018, Zaragoza, Spain. \\ Tel. +34-976762707, e-mail: acecheni@unizar.es
}

\begin{abstract}
Lumbar pain is one of the most common problems of population. Far too often it is caused by ageing and degeneration of intervertebral discs. Fusion techniques, as arthrodesis which used screw fixation, were the first surgeries used to avoid lumbar pathologies. However, arthrodesis reduced dramatically the spine movement.
\end{abstract}

Stand-alone cage is a minimally invasive surgery alternative to lumbar fusion with posterior fixation. Despite their previous reported successful results (Ahmadian et al., 2014) some physicians continue questioning their effectiveness because of the risk of spine destabilization and cage migration (Oxland et al., 2000).

The purpose of this research was to demonstrate that stand-alone cages introduced in a minimally invasive way are a good surgical solution for the IVD diseases.

This main goal has been divided into three different partial goals: prove spinal stability, show the decompression on the neural region and compare the effects over the adjacent discs with and without posterior fixation. It is also of the interest of this work to compare between cage designs based on the above mentioned criteria.

\section{Materials and methods}

A whole L1-S1 lumbar spine intact model validated in previous works (Cegoñino et al., 2014) was simulated under different load conditions. Adding modifications to this model four different surgeries were mimicked as can be seen in Figure I.

Two cage designs were implemented, one bean shaped cage and two parallel piece cage, both made of PEEK. They were placed in the L4-L5 interbody space in stand-alone construct and supplemented with posterior screw fixation.
The criteria used to evaluate the surgery effectiveness were segment stiffness, nerve root decompression, effect over adjacent segments and risk of cage subsidence

\section{Results}

The results showed that stand-alone cages allowed a wider segment movement than screw fixations without compromising the spine stability. The trend of segment stiffness was in accordance with other models (Choi et al., 2013), it was much higher for fixation models than for stand-alone cages

Moreover, it is known that loss of motion in the surgical segment causes long-term adjacent segment disease due to a stress distribution alteration in the surrounding tissues, in this work it was observed that the effect over the adjacent segments stresses was higher in case of posterior fixation.

Apart from spinal stability, the purpose of lumbar surgery is to decompress the damaged segment maintaining the disc height. Here no evidence of nerve root compression in stand-alone constructs attending to disc height loss was seen.

On the other hand, cage subsidence and migration are the most common causes of failure in lumbar surgeries. It was seen that the contact area was larger for the one piece cage than for the two parallel cages, so the contact pressure and shear stresses were more pronounced for the last one which could increase the risk of subsidence.

In addition, there was no evidence that slid relative displacements may cause migration because their magnitude was in the order of micrometers. Instead of that it could be elucidated that the micromovements might stimulate tissue growth.

\section{Conclusions}

To conclude, this work showed that stand-alone cages could be a minimally invasive alternative to 
the posterior fixation devices which caused a dramatically loss of motion in the affected segment involving changes in adjacent discs. It is also possible to discuss that one piece cage may be a better option than two pieces cage because of the more suitable geometry that supplies a largest contact area reducing the risk of subsidence and minimizing the effects over the adjacent segments.

\section{Figures}
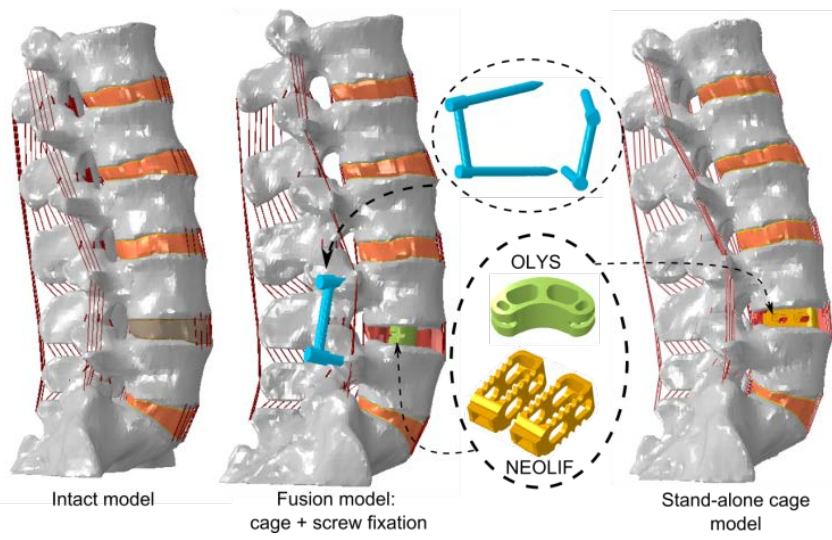

Figure 1. Finite element models of the lumbar spine.

\section{References}

[1]. AHMADIAN, Amir, et al. Stand-Alone Minimally Invasive Lateral Lumbar Interbody Fusion: Multicenter Clinical Outcomes. Journal of Clinical Neuroscience. 2014, 22(4), 740-746.

[2]. CEGOÑINO, J., et al. A Constitutive Model for the Annulus of Human Intervertebral Disc: Implications for Developing a Degeneration Model and its Influence on Lumbar Spine Functioning. Journal of Applied Mathematics. 2014, 2014, 1-15. Avalaible from: doi:10.1155/2014/658719.

[3]. CHOI, KC, et al. Biomechanical Comparison of Anterior Lumbar Interbody Fusion: Stand-Alone Interbody Cage Versus Interbody Cage with Pedicle Screw Fixation - a Finite Element Analysis. BMC Musculoskeletal Disorders. 2013, 14. Avalaible from: doi:10.1186/1471-2474-14-220.

[4]. OXLAND, TR; and LUND, T. Biomechanics of Stand-Alone Cages and Cages in Combination with Posterior Fixation: A Literature Review. European Spine Journal. 2000, 9(Suppl 1), 95-101. 